JOURNAL OF

FUNCTION SPACES AND APPLICATIONS

Volume 7, Number 1 (2009), 33-41
(C) 2009, Scientific Horizon http://www.jfsa.net

\title{
A Beurling-Helson type theorem for modulation spaces
}

\author{
Kasso A. Okoudjou
}

(Communicated by Hans Feightinger)

2000 Mathematics Subject Classification. Primary 42B15; Secondary 42A45, 42B35.

Keywords and phrases. Beurling-Helson theorem, changes of variables, Feichtinger algebra, Fourier multipliers, modulation spaces, Sjöstrand algebra.

Abstract. We prove a Beurling-Helson type theorem on modulation spaces. More precisely, we show that the only $\mathcal{C}^{1}$ changes of variables that leave invariant the modulation spaces $\mathcal{M}^{p, q}\left(\mathbb{R}^{d}\right)$ are affine functions on $\mathbb{R}^{d}$. A special case of our result involving the Sjöstrand algebra was considered earlier by A. Boulkhemair.

\section{Introduction}

Given a function $\phi$ defined from the torus $\mathbb{T}$ to itself, let $\phi^{*}$ be the change of variables defined by

$$
\phi^{*}(u)=u \circ \phi
$$

for any function $u$ defined on $\mathbb{T}$.

In 1953, A. Beurling and H. Helson proved that if $\phi$ is continuous from $\mathbb{T}$ into itself and if $\phi^{*}$ is a bounded linear operator on the Fourier algebra $A(\mathbb{T})=A_{1}(\mathbb{T})$ of absolutely convergent Fourier series, then necessarily $\phi(t)=k t+\phi(0)$ for some $k \in \mathbb{Z}[1]$. The proof of this result involved 
some nontrivial arithmetical considerations. A different proof was given by J.-P. Kahane [15]. The Beurling-Helson theorem was later extended to the higher dimensional setting by W. M. Self [17]. More recently, V. Lebedev and A. Olevskir [16] further extended and generalized the Beurling-Helson theorem. In particular, for $d \geq 1$ and $1 \leq p<\infty$ let $A_{p}\left(\mathbb{R}^{d}\right)=\mathcal{F} L^{p}\left(\mathbb{R}^{d}\right)$ equipped with the norm $\|f\|_{A_{p}}=\|\hat{f}\|_{L^{p}}$ where $\mathcal{F}$ is the Fourier transform defined by $\mathcal{F} f(\omega)=\hat{f}(\omega)=\int_{\mathbb{R}^{d}} f(t) e^{-2 \pi i t \cdot \omega} d t$. It was proved in [16] that if $\phi: \mathbb{R}^{d} \rightarrow \mathbb{R}^{d}$ is $\mathcal{C}^{1}$, and if $\phi^{*}$ maps $A_{p}\left(\mathbb{R}^{d}\right)$ into itself for some $1 \leq p<\infty$, $p \neq 2$, then $\phi(x)=A x+\phi(0)$ where $A$ is a real invertible $d \times d$ matrix. In this higher dimensional setting, the case $p=1$ was already proved in [17]. Observe that since $A_{2}\left(\mathbb{R}^{d}\right)=\mathcal{F} L^{2}\left(\mathbb{R}^{d}\right)=L^{2}\left(\mathbb{R}^{d}\right)$, the class of functions $\phi$ such that $\phi^{*}$ is bounded on $A_{2}\left(\mathbb{R}^{d}\right)$ is quite large. For instance, for any homeomorphism $\phi$ on $\mathbb{T}$ such that $\phi^{-1}$ satisfies the Lipschitz condition, $\phi^{*}$ is bounded on $A_{2}(\mathbb{T})$, and a transference argument can be used to prove similar result for $A_{2}\left(\mathbb{R}^{d}\right)$.

In this note, we shall characterize the $\mathcal{C}^{1}$ changes of variables that leave invariant the modulation spaces (to be defined below). In particular, our result applies to a special subspace of the Fourier algebra called Feichtinger algebra. This space denoted $S_{0}$ was introduced by H. Feichtinger [5] and is the smallest Banach algebra that is invariant under both the translation and the modulation operators. Moreover, the Feichtinger algebra is an example of a modulation space and plays an important role in the theory Gabor frames [11]. In fact, the modulation spaces have also been playing an increasing role in the analysis of pseudodifferential operators $[12,13,20]$. Furthermore, a Banach algebra of pseudodifferential operators known as the Sjöstrand algebra, denoted $S_{\omega}$, and which contains the Hörmander class $S_{0,0}^{0}$, was introduced independently by Feichtinger [6] and J. Sjöstrand [18]. This space is yet another example of a modulation space. We refer to [7] for an updated version of [6] which contains some historical perspectives on the modulation spaces. In 1997, A. Boulkhemair [4] proved that if $\phi$ is a $\mathcal{C}^{1}$ mapping on $\mathbb{R}^{d}$ such that $\phi^{*}$ maps $S_{\omega}$ into itself, then $\phi$ must be an affine function: This is a Beurling-Helson type theorem for the Sjöstrand algebra. It is therefore natural to seek a characterization of the changes of variables that leave invariant modulation spaces. The goal of this note is to extend and generalize this Beurling-Helson type theorem to all the modulation spaces. The main argument in the proof of our result is the fact that the intersection of a modulation space with the space of functions with compact support coincides with the subspace of compactly supported functions in $A_{p}\left(\mathbb{R}^{d}\right)=\mathcal{F} L^{p}\left(\mathbb{R}^{d}\right)$. The proof of this fact as well as the definition of the modulation spaces will be given in Section 2. Finally, in 
Section 3 we shall prove our main result. In the sequel, we shall denote by $|A|$ the Lebesgue measure of a measurable subset $A$ of $\mathbb{R}^{d}$.

\section{Preliminaries}

2.1 Modulation spaces. The Short-Time Fourier Transform (STFT) of a function $f$ with respect to a window $g$ is

$$
V_{g} f(x, y)=\int_{\mathbb{R}} f(t) \overline{g(t-x)} e^{-2 \pi i y t} d t
$$

whenever the integral makes sense. This definition can be extended to $f \in \mathcal{S}^{\prime}\left(\mathbb{R}^{d}\right)$ and $g \in \mathcal{S}\left(\mathbb{R}^{d}\right)$ and yields a continuous function $V_{g} f$, see [11].

Definition 1. Given $1 \leq p, q \leq \infty$, and given a window function $0 \neq g \in \mathcal{S}$, the modulation space $\mathcal{M}^{p, q}=\mathcal{M}^{p, q}\left(\mathbb{R}^{d}\right)$ is the space of all distributions $f \in \mathcal{S}^{\prime}$ for which the following norm is finite:

$$
\|f\|_{\mathcal{M}^{p, q}}=\left(\int_{\mathbb{R}^{d}}\left(\int_{\mathbb{R}^{d}}\left|V_{g} f(x, y)\right|^{p} d x\right)^{q / p} d y\right)^{1 / q}
$$

with the usual modifications if $p$ and/or $q$ are infinite.

Remark 1. The definition is independent of the choice of the window $g$ in the sense of equivalent norms.

The modulation spaces were originally introduced by Feichtinger [6]. We refer to [11] and the references therein for more details about modulation spaces.

The Feichtinger algebra $S_{0}$ which coincides with the modulation space $\mathcal{M}^{1,1}\left(\mathbb{R}^{d}\right)$ is a Banach algebra under both pointwise multiplication and convolution. Furthermore, $\mathcal{M}^{1,1}\left(\mathbb{R}^{d}\right)$ like $\mathcal{M}^{p, p}\left(\mathbb{R}^{d}\right) 1 \leq p \leq \infty$ is invariant under the Fourier transform $[5,8,9]$.

While the Beurling-Helson theorem completely classifies the changes of variables that operate in $A_{1}(\mathbb{T})$ (and also on $A_{1}(\mathbb{R})$ ) it was still unknown what changes of variables operate on the Feichtinger algebra and more generally on the modulation spaces. This question will be completely settled below.

2.2 Local modulation spaces. The theory of modulation can be defined in the general setting of locally compact Abelian groups [7]. In particular, it can be shown that for $G=\mathbb{Z}^{d}$ (or any discrete group), $\mathcal{M}^{p, q}(G)=\ell^{p}(G)$. Similarly, if $G=\mathbb{T}^{d}$ (or any compact group), 
$\mathcal{M}^{p, q}(G)=\mathcal{F} L^{q}(G)$. Here we focus on functions that are locally in a modulation space.

In the sequel we shall denote by $\mathcal{M}_{\text {comp }}^{p, q}\left(\mathbb{R}^{d}\right)$ the subspace of $\mathcal{M}^{p, q}\left(\mathbb{R}^{d}\right)$ consisting of compactly supported functions, and by $\mathcal{M}_{\text {loc }}^{p, q}\left(\mathbb{R}^{d}\right)$ the space of functions that are locally in $\mathcal{M}^{p, q}\left(\mathbb{R}^{d}\right)$. In particular, $u \in \mathcal{M}_{\text {loc }}^{p, q}\left(\mathbb{R}^{d}\right)$ if and only if for each $g \in \mathcal{C}_{0}^{\infty}\left(\mathbb{R}^{d}\right)$ with $\operatorname{supp}(g) \subset K$ where $K$ is a compact subset of $\mathbb{R}^{d}$, we have $u_{K}=g u \in \mathcal{M}^{p, q}\left(\mathbb{R}^{d}\right)$, i.e., $u_{K} \in \mathcal{M}_{\text {comp }}^{p, q}\left(\mathbb{R}^{d}\right)$.

$\left(\mathcal{F} L^{q}\right)_{c o m p}\left(\mathbb{R}^{d}\right)$ and $\left(\mathcal{F} L^{q}\right)_{l o c}\left(\mathbb{R}^{d}\right)$ are defined similarly.

The next result contains the key argument in the proof of our main result. We wish to point out that some special cases of the result are already known. For instance, the result was proved for $\mathcal{M}^{\infty, 1}\left(\mathbb{R}^{d}\right)$ in [4, Theorem 5.1], while [9] dealt with $\mathcal{M}^{p, p}\left(\mathbb{R}^{d}\right) 1 \leq p<\infty$. Furthermore, an independent and different proof of part b. of Lemma 1 using convolution relations on generalized amalgam spaces was indicated to us by H. Feichtinger [10].

Lemma 1. Let $1 \leq p, q \leq \infty$. Then the following statements hold
a. $\mathcal{M}_{\text {comp }}^{p, q}\left(\mathbb{R}^{d}\right)=\left(\mathcal{F} L^{q}\right)_{\text {comp }}\left(\mathbb{R}^{d}\right)$.
b. $\mathcal{M}_{\text {loc }}^{p, q}\left(\mathbb{R}^{d}\right)=\left(\mathcal{F} L^{q}\right)_{l o c}\left(\mathbb{R}^{d}\right)$.

Proof. We shall only prove part a. of the result as part b. follows from the definition of $\mathcal{M}_{l o c}^{p, q}\left(\mathbb{R}^{d}\right)$. Furthermore, to prove a. it suffices to show that given a compact subset $K$ of $\left.\mathbb{R}^{d} \mathcal{M}^{p, q}\left(\mathbb{R}^{d}\right)\right|_{K}=\left.\mathcal{F} L^{q}\left(\mathbb{R}^{d}\right)\right|_{K}$. Note that this last equation holds not only as set equality, but also as equality of Banach spaces with equivalent norms.

Let $R>0$ be given and let $u \in \mathcal{F} L^{q}\left(\mathbb{R}^{d}\right)$ such that $\operatorname{supp}(u) \subset B_{R}(0)$. Let $g \in \mathcal{C}_{c}^{\infty}\left(\mathbb{R}^{d}\right)$ with $\operatorname{supp}(g) \subset B_{R}(0)$. Then, for each $\omega \in \mathbb{R}^{d}$, $V_{g} u(\cdot, \omega)$ is supported in $B_{2 R}(0)$. Thus, using the fact that $\left|V_{g} u(x, \omega)\right|=$ $\left|V_{\hat{g}} \hat{u}(\omega,-x)\right|=\left|\mathcal{F}^{-1}\left(\hat{u} \cdot T_{\omega} \overline{\hat{g}}\right)(x)\right|$ we have the following estimates

$$
\begin{aligned}
\left\|V_{g} u(\cdot, \omega)\right\|_{L^{p}} & \leq\left|B_{2 R}(0)\right|^{1 / p}\left\|V_{g} u(\cdot, \omega)\right\|_{L^{\infty}} \\
& =\left|B_{2 R}(0)\right|^{1 / p}\left\|\mathcal{F}^{-1}\left(\hat{u} \cdot T_{\omega} \overline{\hat{g}}\right)\right\|_{L^{\infty}} \\
& \leq\left|B_{2 R}(0)\right|^{1 / p}\left\|\hat{u} \cdot T_{\omega} \overline{\hat{g}}\right\|_{L^{1}} \\
& \leq\left|B_{2 R}(0)\right|^{1 / p}|\hat{u}| *|\hat{g}|(\omega) .
\end{aligned}
$$

Consequently, $\left\|V_{g} u\right\|_{L^{p, q\left(\mathbb{R}^{2 d}\right)}} \leq\left|B_{2 R}(0)\right|^{1 / p}\|\hat{u}\|_{L^{q}\left(\mathbb{R}^{d}\right)}\|\hat{g}\|_{L^{1}\left(\mathbb{R}^{d}\right)}$, that is

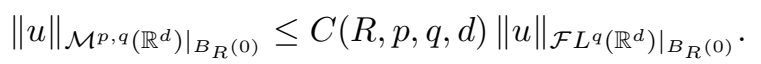

Thus,

$$
\left.\left.\mathcal{F} L^{q}\left(\mathbb{R}^{d}\right)\right|_{B_{R}(0)} \subset \mathcal{M}^{p, q}\left(\mathbb{R}^{d}\right)\right|_{B_{R}(0)}
$$


For the converse, let $R>0$ be given and $u \in \mathcal{M}^{p, q}\left(\mathbb{R}^{d}\right)$ such that $\operatorname{supp}(u) \subset B_{R}(0)$. Let $g \in \mathcal{C}_{0}^{\infty}\left(\mathbb{R}^{d}\right)$ such that $g \equiv 1$ on $B_{2 R}(0)$. It is trivially seen that for all $x \in B_{R}(0)$ and for all $t \in B_{R}(0), g(t-x)=1$. Thus, for all $\omega \in \mathbb{R}^{d}$ and for $x \in B_{R}(0)$,

$$
\begin{aligned}
\hat{u}(\omega) \chi_{B_{R}(0)}(x) & =\chi_{B_{R}(0)}(x) V_{g} u(x, \omega) \\
& =\chi_{B_{R}(0)}(x) \int_{B_{R}(0)} u(t) e^{-2 \pi i t \cdot \omega} \overline{g(t-x)} d t .
\end{aligned}
$$

Therefore,

$$
\left|B_{R}(0)\right|^{1 / p}|\hat{u}(\omega)|=\left\|\chi_{B_{R}(0)}(\cdot) V_{g} u(\cdot, \omega)\right\|_{L^{p}} .
$$

Hence, $\|\hat{u}\|_{L^{q}} \leq\left|B_{R}(0)\right|^{-1 / p}\left\|V_{g} u\right\|_{L^{p, q}}$, that is

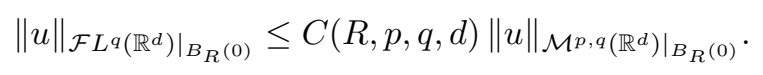

Therefore, $\left.\left.\mathcal{M}^{p, q}\left(\mathbb{R}^{d}\right)\right|_{B_{R}(0)} \subset \mathcal{F} L^{q}\left(\mathbb{R}^{d}\right)\right|_{B_{R}(0)}$. We can now conclude that

$$
\left.\mathcal{M}^{p, q}\left(\mathbb{R}^{d}\right)\right|_{B_{R}(0)}=\left.\mathcal{F} L^{q}\left(\mathbb{R}^{d}\right)\right|_{B_{R}(0)} .
$$

\section{Main results}

Before stating our main result, we wish to indicate that it is trivially seen that all the modulation spaces are invariant under affine changes of variables. That is, let $1 \leq p, q \leq \infty$ and $\phi: \mathbb{R}^{d} \rightarrow \mathbb{R}^{d}$ be an affine mapping, i.e., $\phi(x)=A x+b$ where $A$ is a $d \times d$ real invertible matrix and $b \in \mathbb{R}^{d}$. Then the linear operator $\phi^{*}$ given by (1) maps $\mathcal{M}^{p, q}\left(\mathbb{R}^{d}\right)$ into itself, that is

$$
\phi^{*}\left(\mathcal{M}^{p, q}\left(\mathbb{R}^{d}\right)\right) \subset \mathcal{M}^{p, q}\left(\mathbb{R}^{d}\right) .
$$

Indeed, let $g \in \mathcal{S}$ and $u \in \mathcal{M}^{p, q}\left(\mathbb{R}^{d}\right)$, and $\tilde{g}=g \circ A^{-1}$ where $A^{-1}$ is the inverse of $A$. The result follows from

$$
V_{g} \phi^{*}(u)(x, \omega)=\frac{1}{|\operatorname{det} A|} e^{-2 \pi i \omega \cdot A^{-1} b} V_{\tilde{g}} u\left(A x+b,\left(A^{*}\right)^{-1} \omega\right)
$$

where $A^{*}$ denote the conjugate of $A$.

If we restrict our attention to the modulation spaces $\mathcal{M}^{p, p}\left(\mathbb{R}^{d}\right) 1<p<$ $\infty$ the following stronger result can be proved. For Proposition 1 we assume that $\mathbb{R}^{d}=\cup_{k=1}^{N} Q_{k}$ where for each $k, Q_{k}$ is a (possible infinite) "cube" 
with sides parallel to the coordinates axis. Moreover, we assume that for $k=1, \ldots, N$ the $Q_{k}$ s have disjoint interiors.

Proposition 1. Let $\phi$ be a continuous on $\mathbb{R}^{d}$ such that for $k=1, \ldots, N$, the restriction $\phi_{k}$ of $\phi$ to $Q_{k}$ is an affine function given by $\phi_{k}(x)=A_{k} x+b_{k}$ where $A_{k}$ is a real invertible $d \times d$ matrix and $b_{k} \in \mathbb{R}^{d}$. Then the linear operator $\phi^{*}$ given by (1) maps $\mathcal{M}^{p, p}\left(\mathbb{R}^{d}\right)$ into itself, that is

$$
\phi^{*}\left(\mathcal{M}^{p, p}\left(\mathbb{R}^{d}\right)\right) \subset \mathcal{M}^{p, p}\left(\mathbb{R}^{d}\right) .
$$

Proof. It is evident from the definition of the modulation spaces that $\mathcal{M}^{p, p}$ is invariant under the Fourier transform, see $[8,9]$. Let $u \in \mathcal{M}^{p, p}\left(\mathbb{R}^{d}\right)$, then

$$
\phi^{*}(u)=u \circ \phi=\sum_{k=1}^{N} \chi_{Q_{k}} \cdot(u \circ \phi)=\sum_{k=1}^{N} \chi_{Q_{k}} \cdot\left(u \circ \phi_{k}\right),
$$

and so

$$
\left\|\phi^{*}(u)\right\|_{\mathcal{M}^{p, p}} \leq \sum_{k=1}^{N}\left\|\chi_{Q_{k}} \cdot\left(u \circ \phi_{k}\right)\right\|_{\mathcal{M}^{p, p}} .
$$

As indicated above, $u \circ \phi_{k} \in \mathcal{M}^{p, p}$. Hence, $v_{k}=\mathcal{F}^{-1}\left(u \circ \phi_{k}\right) \in \mathcal{M}^{p, p}\left(\mathbb{R}^{d}\right)$ as well. Moreover, note that $\chi_{Q_{k}}$ is a bounded Fourier multiplier on all $\mathcal{M}^{p, p}\left(\mathbb{R}^{d}\right)$ : this follows from $[2$, Theorem 1$]$ in the case $d=1$, and from [3, Theorem 6] when $d>1$. Consequently, using the invariance of $\mathcal{M}^{p, p}\left(\mathbb{R}^{d}\right)$ under the Fourier transform, we conclude that there exists $c_{k}>0$ such that

$$
\left\|\chi_{Q_{k}} \cdot\left(u \circ \phi_{k}\right)\right\|_{\mathcal{M}^{p, p}}=\left\|\mathcal{F}^{-1}\left(\chi_{Q_{k}} \cdot \hat{v}_{k}\right)\right\|_{\mathcal{M}^{p, p}} \leq c_{k}\|u\|_{\mathcal{M}^{p, p}},
$$

from which the proof follows.

Remark 2. The conclusion of Proposition 1 holds if we used an infinite decomposition of $\mathbb{R}^{d}$, that is if we assume that $\mathbb{R}^{d}=\cup_{k=1}^{\infty} Q_{k}$ where the cubes $Q_{k}$ still have sides parallel to the coordinate axis and disjoint interiors. In this case, the extra assumption needed to prove the previous result is that the constants $c_{k}$ appearing in the above proof, are uniformly bounded, i.e., $\sup _{k} c_{k}<\infty$.

We are now ready to state and prove our main result.

Theorem 1. Let $\phi: \mathbb{R}^{d} \rightarrow \mathbb{R}^{d}$ be a $\mathcal{C}^{1}$ function. Assume that the operator $\phi^{*}$ defined by (1) maps $\mathcal{M}^{p, q}\left(\mathbb{R}^{d}\right)$ into itself, i.e., $\phi^{*}\left(\mathcal{M}^{p, q}\left(\mathbb{R}^{d}\right)\right) \subset$ $\mathcal{M}^{p, q}\left(\mathbb{R}^{d}\right)$ for some $1 \leq p, q \leq \infty$, with $2 \neq q<\infty$. Then $\phi$ is an affine mapping, that is $\phi(x)=A x+\phi(0)$ for some real invertible $d \times d$ matrix $A$.

In particular, the Feichtinger algebra $\mathcal{M}^{1,1}\left(\mathbb{R}^{d}\right)$ is preserved by, and only by affine changes of variables. 
Proof. Because $\phi^{*}\left(\mathcal{M}^{p, q}\left(\mathbb{R}^{d}\right)\right) \subset \mathcal{M}^{p, q}\left(\mathbb{R}^{d}\right)$ and $\phi^{*}(u)=u \circ$ $\phi$ is compactly supported whenever $u$ is, Lemma 1 implies that $\phi^{*}$ maps $\mathcal{M}_{\text {comp }}^{p, q}\left(\mathbb{R}^{d}\right)=\left(\mathcal{F} L^{q}\right)_{\text {comp }}\left(\mathbb{R}^{d}\right)$ into itself as well as $\mathcal{M}_{\text {loc }}^{p, q}\left(\mathbb{R}^{d}\right)=$ $\left(\mathcal{F} L^{q}\right)_{l o c}\left(\mathbb{R}^{d}\right)$ into itself. Therefore,

when $d=1$ and $q=1$, the Beurling-Helson Theorem [1, pp. 84-86], implies that $\phi(x)=a x+\phi(0)$;

when $d=1$ and $1<q<\infty, q \neq 2$, it follows from [16, Theorem 3] that $\phi(x)=a x+\phi(0)$;

when $d>1$ and $q=1$, it follows from [17, Corollary 1] that $\phi(x)=$ $A x+\phi(0)$, where $A$ is a real invertible $d \times d$ matrix;

when $d>1$ and $1<q<\infty, q \neq 2$, it follows from [16, Theorem 6] that $\phi(x)=A x+\phi(0)$, where $A$ is a real invertible $d \times d$ matrix.

Remark 3. The fact that $q \neq 2$ in Theorem 1 was justified in the Introduction. Moreover, we restricted to $q<\infty$, because the key ingredients in the proof of our main result are [16, Theorem 3, Theorem $6]$ whose proofs are based on a density argument. It is not clear to us if Theorem 1 holds for $q=\infty$.

Remark 4. Using Lemma 1 and [16, pp. 214], it follows that if $\phi$ : $\mathbb{R}^{d} \rightarrow \mathbb{R}^{d}$ is nonlinear and $\mathcal{C}^{2}$, then $\phi^{*}$ is not bounded on $\mathcal{M}^{p, q}$. This fact together with Proposition 1, show that the $\mathcal{C}^{1}$ condition in Theorem 1 is the only nontrivial smoothness condition to impose on $\phi$.

For the Sjöstrand algebra $S_{\omega}$ which coincides with the modulation space $\mathcal{M}^{\infty, 1}\left(\mathbb{R}^{d}\right)$, Theorem 1 was proved in under a weaker assumption on $\phi$. More specifically, it was proved in [4, Theorem 5.1] that if $\phi$ is a proper mapping, i.e., $\phi$ is continuous on $\mathbb{R}^{d}$ and $\phi^{-1}(K)$ is a compact set for any compact subset $K$ of $\mathbb{R}^{d}$, and if $\phi^{*}\left(\mathcal{M}^{\infty, 1}\left(\mathbb{R}^{d}\right)\right) \subset \mathcal{M}^{\infty, 1}\left(\mathbb{R}^{d}\right)$ then $\phi(x)=A x+\phi(0)$. It is also straightforward to prove Theorem 1 under this weaker assumption on $\phi$.

Finally, we wish to conclude this paper by pointing out the connection of our main result to certain Fourier multipliers. More precisely, let $\sigma$ be a function defined on $\mathbb{R}^{d}$. The Fourier multiplier with symbol $\sigma$ is the operator $H_{\sigma}$ initially defined on $\mathcal{S}$ by

$$
H_{\sigma} f(x)=\int_{\mathbb{R}^{d}} \sigma(\xi) \hat{f}(\xi) e^{2 \pi i \xi \cdot x} d \xi .
$$

We refer to [19] for more on Fourier multipliers. As mentioned above, there is a strong connection between the $L^{p}$-continuity of the Fourier multipliers and the Beurling-Helson theorem. In particular, the family of homomorphisms $e^{i \phi(\xi)}$ on the space of $L^{p}$-Fourier multipliers was investigated by Hörmander in [14, Section 1.3]. It is easily seen that 
$\sigma_{0}(\xi)=e^{i \xi}$, then $H_{\sigma_{0}}$ is bounded on all $L^{p}\left(\mathbb{R}^{d}\right)$ for $1 \leq p \leq \infty$ and $d \geq 1$. Hörmander proved that if $\phi: \mathbb{R}^{d} \rightarrow \mathbb{R}^{d}$ is $\mathcal{C}^{2}$ and if $\phi^{*}\left(\sigma_{0}\right)(\xi)=\sigma_{0}(\phi(\xi))=e^{i \phi(\xi)}$ is a bounded Fourier multiplier on $L^{p}\left(\mathbb{R}^{d}\right)$ for some $1<p<\infty$ and $p \neq 2$, then $\phi$ is an affine function [14, Theorem 1.15]. It is interesting to note that there exist nonlinear (nonaffine) functions $\phi$ on $\mathbb{R}^{d}$ such that the Fourier multipliers with symbols $\phi^{*}\left(\sigma_{0}\right)(\xi)=\sigma_{0}(\phi(\xi))=e^{i \phi(\xi)}$ are bounded on all modulation spaces [3].

Acknowledgment. The author would like to thank Chris Heil for bringing some of the questions discussed in this work to his attention. He also thanks Árpád Bényi, Karlheinz Gröchenig, Norbert Kaiblinger, and Luke Rogers for helpful discussions. Finally, the author is grateful to Hans Feichtinger

for pointing out numerous realted results as well as for many comments on earlier versions of this note.

\section{References}

[1] A. Beurling and H. Helson, Fourier-Stieltjes transform with bounded powers, Math. Scand., 1 (1953), 120-126.

[2] A. Bényi, L. Grafakos, K. Gröchenig, and K. Okoudjou, A class of Fourier multipliers for modulation spaces, Appl. Comput. Harmon. Anal., 19 (2005), 131-139.

[3] A. Bényi, K. Gröchenig, K.A. Okoudjou, and L. G. Rogers, Unimodular Fourier multipliers for modulation spaces, J. Funct. Anal., 246 (2007), 366-384.

[4] A. Boulkhemair, Remarks on a Wiener type pseudodifferential algebra and Fourier integral operators, Math. Res. Lett., 4 (1997), 53-67.

[5] H.G. Feichtinger, On a new Segal algebra, Monatsh. Math., 92 (1981), 269-289.

[6] H.G. Feichtinger, Modulation spaces on locally Abelian groups, Technical report, University of Vienna, January 1983.

[7] H.G. Feichtinger, Modulation spaces on locally Abelian groups, in Proc. Internat. Conf. on Wavelets and Applications (Chennai, 2002), R. Radha, M. Krishna, S. Thangavelu (eds.), New Delhi Allied Publishers (2003), p.1-56

[8] H.G. Feichtinger, Atomic characterization of modulation spaces through Gabor-type representations, in: Proc. Conf. Constructive Function Theory, Rocky Mountain J. Math., 19 (1989), 113-126.

[9] H.G. Feichtinger, Generalized amalgams, with applications to Fourier transform, Can. J. Math., 42 (1990), 395-409. 
[10] H.G. Feichtinger, Personal communication, 2007.

[11] K. Gröchenig, Foundations of Time-Frequency Analysis, Birkhäuser, Boston, 2001.

[12] K. Gröchenig and C. Heil, Modulation spaces and pseudodifferential operators, Int. Eq. Oper. Theory, 34 (1999), 439-457.

[13] C. Heil, Integral operators, pseudodifferential operators, and Gabor frames, in: "Advances in Gabor Analysis," H. G. Feichtinger and T. Strohmer, eds., Birkhäuser, Boston (2003), 153-169.

[14] L. Hörmander, Estimates for translation invariant operators in $L^{p}$ spaces, Acta Math., 104 (1960), 93-140.

[15] J.-P. Kahane, Séries de Fourier Absolument Convergentes, SpringerVerlag, New York, 1970.

[16] V. Lebedev and A. Olevskiǐ, $C^{1}$ changes of variable: Beurling-Helson type theorem and Hörmander conjecture on Fourier multipliers, Geom. Funct. Anal., 4 (1994), 213-235.

[17] W.M. Self, Some consequences of the Beurling-Helson theorem, Rocky Mountain J. Math., 6 (1976), 177-180.

[18] J. Sjöstrand, An algebra of pseudodifferential operators, Math. Res. Lett., 1 (1994), 185-192.

[19] E.M. Stein, Singular Integrals and Differentiability Properties of Functions, Princeton Mathematical Series, Princeton University Press, 1970.

[20] J. Toft, Continuity properties for modulation spaces, with applications to pseudodifferential operators. I, J. Funct. Anal., 207 (2004), 399-429.

Department of Mathematics

University of Maryland

College Park, MD, 20742

USA

(E-mail :kasso@math.umd.edu) 


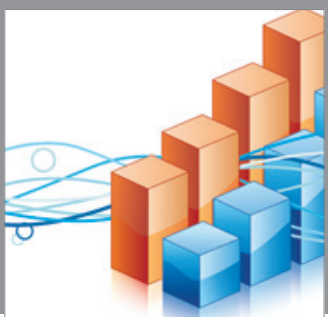

Advances in

Operations Research

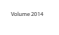

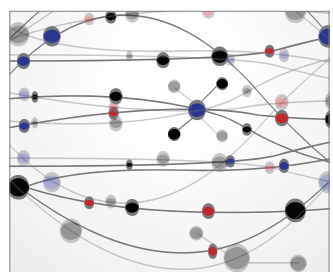

\section{The Scientific} World Journal
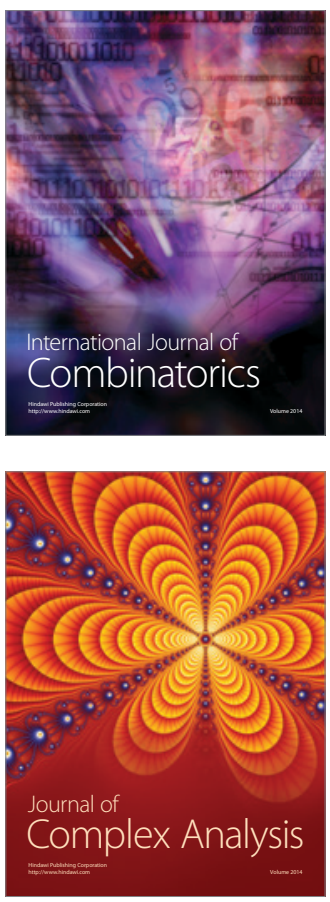

International Journal of

Mathematics and

Mathematical

Sciences
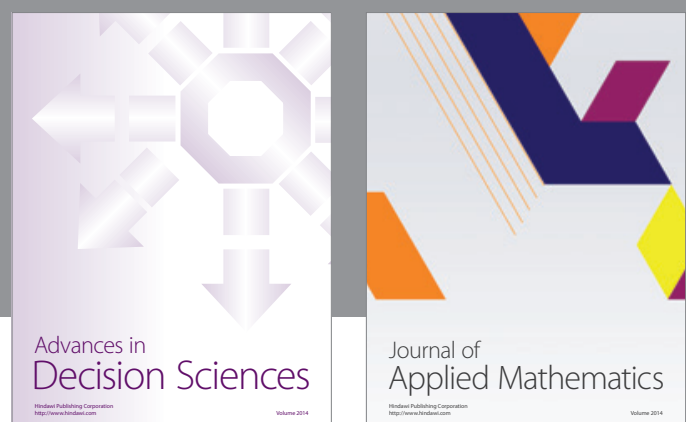

Journal of

Applied Mathematics
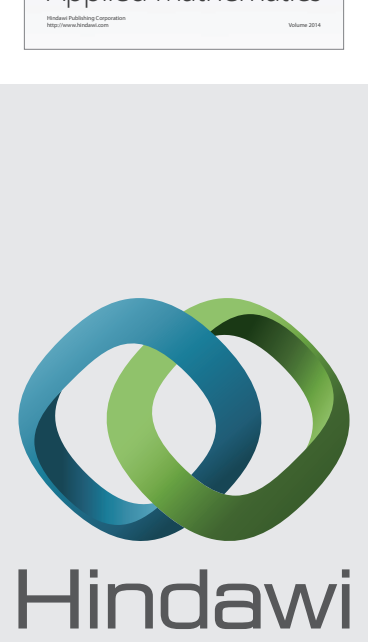

Submit your manuscripts at http://www.hindawi.com
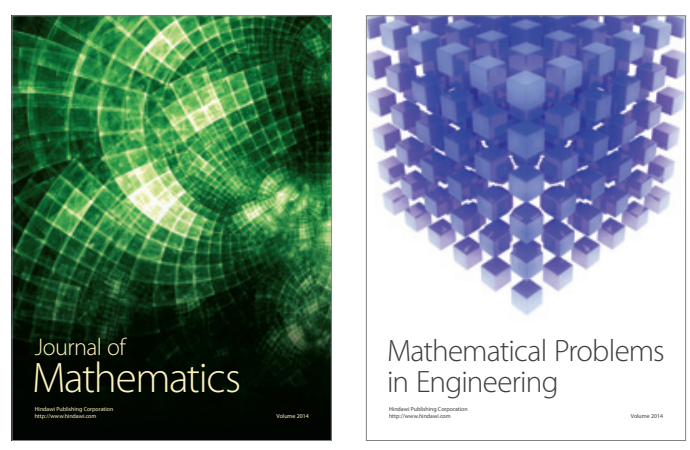

Mathematical Problems in Engineering
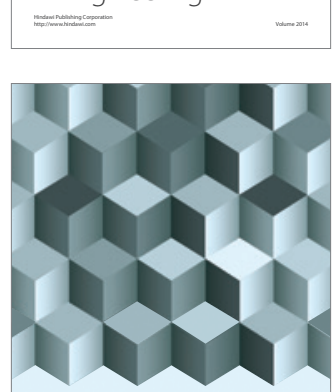

Journal of

Function Spaces
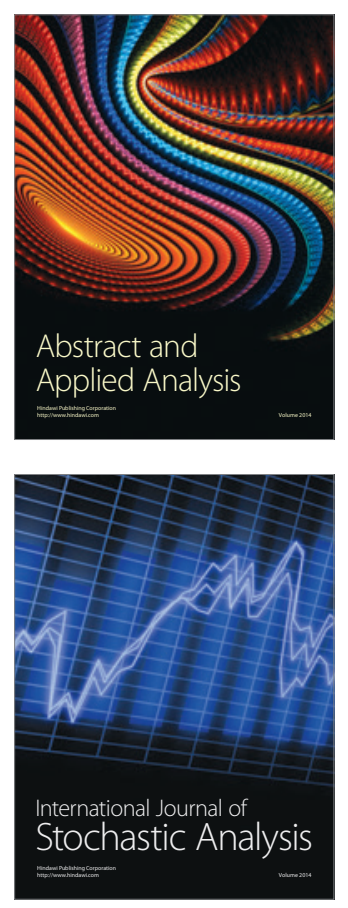

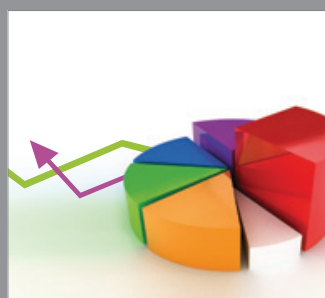

ournal of

Probability and Statistics

Promensencen
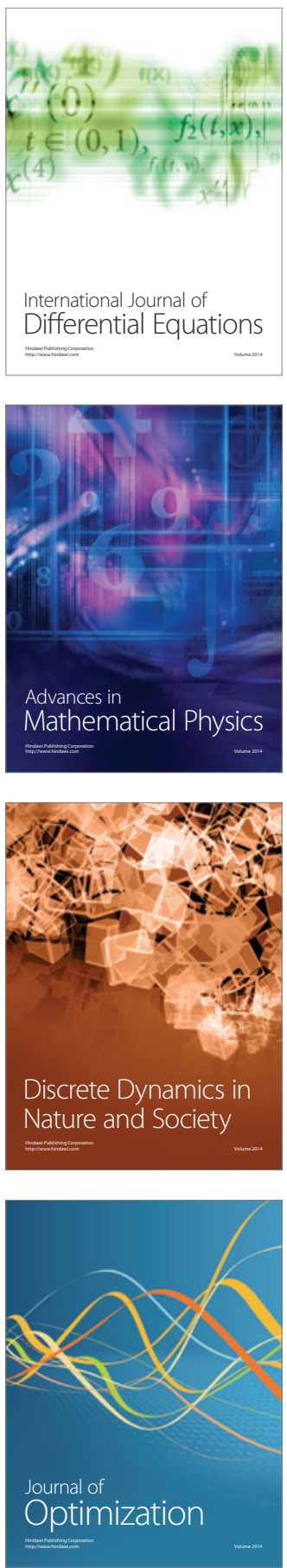